\title{
Pharmacokinetic Concentration Baseline
} Flag

National Cancer Institute

\section{Source}

National Cancer Institute. Pharmacokinetic Concentration Baseline Flag. NCI Thesaurus. Code C119902.

An indication or description that pharmacokinetic concentration data is a baseline value. 